\title{
Risk Evaluation and Risk Control in Road Overbuilding of Transport Routes for Dangerous Goods
}

\author{
Johan Lundin \\ WSP, Stockholm-Globen SE-121 88, Sweden
}

\begin{abstract}
Overbuilding, i.e. capping or decking, of roads assigned for the transportation of dangerous goods in major cities is being considered more often in many countries including Sweden. This type of facility introduces a number of risks and challenges related to rare events, such as dangerous goods accidents in tunnels, with a potential to cause substantial damage, e.g. collapse of buildings on the over site development. Many argue that the major problem associated with risk evaluation of such risks is the lack of commonly agreed acceptance criteria and regulations. Approaching the problem from a scientific perspective suggests that the root cause of this problem has not been adequately identified. However, there are strong indications that it is because the nature of the risk is not adequately addressed in risk analysis. In this study, we have structured the problem and propose ways of making progress in the evaluation of risk exposure and risk control. It is concluded that several challenges are inherent in the risk management of decking over routes for the transport of dangerous goods, and that these must be addressed in order to be able to control the risk. One of the complicating factors is that it is practically and economically impossible to construct the overbuilding so that it can withstand the maximum permissible explosion load. The main conclusions of this study are that overbuilding of dangerous goods routes has the potential to lead to catastrophic events, that the transport of dangerous goods in the future is associated with considerable intrinsic uncertainties, and that the possibility of supervising and controlling the transport of goods is limited once the route is in use.
\end{abstract}

Key words: Capping, overbuilding, dangerous goods, tunnels, categorization, risk management, hazardous materials, transportation.

\section{Introduction}

Dangerous goods are not only encountered in industry, but in other activities, for instance, at petrol stations, sewage treatment plants, construction sites and hospitals. It is necessary to transport dangerous goods to and from these facilities by road or by rail, inducing risks to the environment and to health and safety. For society to accept the transport of such goods, it is important that the public is not exposed to unnecessary or unacceptable risks. The transport of dangerous goods is therefore governed by extensive regulations [1], with requirements that potential risks are considered in the planning of routes or developments adjacent to routes [2-4] in the planning processes. In order to balance the need for transportation of dangerous goods with safety aspects

Corresponding author: Johan Lundin, Ph.D., technical director; research fields: risk analysis, risk management, tunnel safety, and land-use planning. E-mail: johan.lundin@wsp.com. in land use planning, dedicated routes are recommended for the transportation of dangerous goods. Land suitable for development is becoming limited, which means that areas previously not deemed suitable for development due to noise, emissions and other risks associated with heavy traffic, are now being reconsidered. One way of increasing the amount of land is through over site development, e.g. by decking over existing road or rail infrastructure.

Interest in overbuilding has increased considerably in large cities in Sweden in the past decade $[5,6]$, as it is believed to have several advantages within the framework of sustainable city development, such as increased accessibility, increased traffic safety for pedestrians, reduced noise and a general improvement in the urban environment [7]. The principle is illustrated in Fig. 1. However, the main driver is commercial, by enabling densification of urban environments and development of central areas, which 


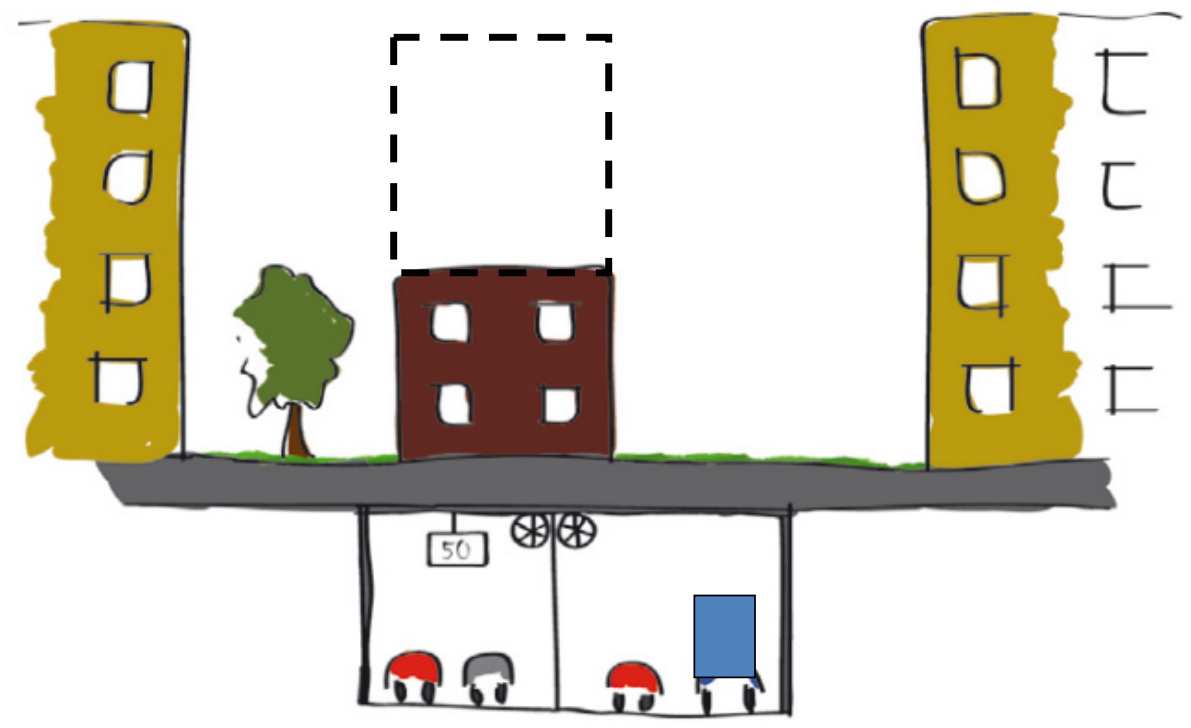

Fig. 1 Illustration of the overbuilding of a major road in an urban environment, allowing for over site development of the areas made available.

is often necessary for financing these major projects. Bearing in mind that there is a housing shortage in many Swedish towns and cities, the possibility of developing new areas is of high interest to local authorities, developers and, not least, those who need somewhere to live. Housing is high up on the Swedish Government's agenda and on the political agenda in general. For example, the City Planning Division in Stockholm is assigned to conduct a survey of places suitable for overbuilding in order to provide new opportunities for development [8].

However, overbuilding is associated with several challenges; for example, the cost of laying foundations and the $\mathrm{CO}_{2}$ footprint are much higher than in other types of construction. Furthermore, a number of safety issues must be addressed. Complicated and extensive agreements are also required between several parties for the financing and execution of the project. Overbuilding is not necessarily regarded as a sustainable practice in urban development. It depends on the perspective from which the project is analysed. However, it is clear that it has the potential to play an important part in sustainable urban development.

One consequence of these challenges is that the presumptions on which the planning of an overbuilding project is based are uncertain. There is a risk that it will not be possible to assess the feasibility of the project, or the suppositions on which the business case is based until a relatively late stage in the planning process. This poses a threat to the whole project as the uncertainties in the return on investments are too great.

One of the greatest project risks in decking over roads has been found to be the issue of safety when the route is used for the transport of dangerous goods. Uncertainty concerning risk evaluation and risk control is a major factor, i.e. how we can ensure that an acceptable level of safety is achieved and maintained over time. The uncertainty is, in fact, so tangible that it may cause a major threat to the feasibility of projects, despite the societal benefits it offers in terms of urban development.

\section{Aims and Objectives}

The aim of this study was to apply scientific principles to investigate the conditions for, and to provide recommendations on, the risk evaluation of overbuilding of routes intended for the transportation of dangerous goods, with the ultimate aim of making risk control possible. The intention is that this study will allow planning to be conducted in a transparent 
and credible way in the early stages of the project, in order to reduce the project risk, and to improve its feasibility. The following approaches were adopted for the study:

- Identification of critical aspects of the nature of risk and risk control associated with overbuilding;

- Development of recommendations regarding the information used to inform decisions makers regarding these risks;

- Clarify the conditions for risk management to ensure risk control over time.

\section{Background}

This section presents a short background on the underlying reasons why the question of safety in the present case is perceived as being so complex that it poses a threat to the feasibility of overbuilding projects. Overbuilding of roads is perceived as problematic by both developers and supervisory authorities, i.e. authorities having jurisdiction. The planning process takes time, and delays resolving the safety issues are common; there is often a lack of transparency, and the level of predictability is low. The way in which authorities deal with the question of safety is sometimes perceived as being arbitrary, which affects the credibility of the planning process and the supervisory authority (AHJ). This is completely contrary to the objectives of the planning and construction processes. The uncertainties in the planning process are highly undesirable from the perspective of the project management and the investors. Examples of these uncertainties are the floor-space index, constructability, the cost, the involvement of the authorities, and final approval.

The problems identified in dealing with safety issues in overbuilding projects from a number of previous projects and in studies of the current knowledge base commissioned by national and regional authorities $[6,7]$ can be broadly summarized as follows: unclear regulations, the lack of a clearly defined acceptable level of risk, indecision on the part of authorities regarding design values of blast, and the fact that it is not financially reasonable to design for scenarios with very low probabilities. None of the above challenges are unique to overbuilding, but they apply in principle to all kinds of risk management. Unfortunately, little progress has been made in this area during the past decades and little or no research initiatives have been taken. We therefore deemed it necessary to investigate the problems more deeply, and structure them, before attempts were made to provide solutions.

Experiences of overbuilding in Europe are limited. According to one of few inventories [9], it appears that overbuilding has only been carried out along routes where the transport of dangerous goods with the potential to affect overlying constructions is not permitted. This simplifies the problem of risk management considerably. In several regions and countries, for example, in many states in the USA, the transport of dangerous goods through highway tunnels close to urban areas is restricted or forbidden. Overbuilding of long road routes on which dangerous goods are transported is a relatively new concept in Sweden. Little empirical data are therefore available regarding both the project as such and accidents. Worth noting is that in Sweden, at least in the area of Stockholm, seems to be less restrictive of these types of overbuilds compared to other countries.

\section{Major Risk-related Problems}

In order to structure the problems and suggest solutions, the risk analytical context in question, which affects both the actual risk and risk management, is described briefly below, based on a literature study and the author's experiences from previous projects.

\subsection{Many Parties and a High Level of Complexity}

Risk management in cases of overbuilding is complicated in many respects as several parties are involved, and the abilities, costs and risks associated with each party are affected by the way in which the risk management strategy for the project is resolved. 
Any project is dependent on several planning processes simultaneously, mainly road planning and physical planning. Knowledge is limited regarding the problems concerning safety and which measures are required to solve or mitigate them. As the incentives, responsibilities and abilities regarding risk management vary among the parties involved, there is a need for coordinated measures and collaborative solutions in order to carry a project. In the case of several public authorities, this may mean a change in role from being a supervisory authority, to becoming an active partner who is required to facilitate the realisation of the project. The greater the number of the parties involved, the greater the complexity of the planning process. Examples of authorities often involved in such projects are various committees in local authorities, e.g. for traffic planning, city planning and development, the emergency services, as well as national and regional authorities such as the Swedish Transport Agency, the Swedish Transport Administration and the relevant County Administrative Board [6]. The need for supervision, approval and consultation is large.

\subsection{Geographical Demarcation}

Problems may arise if the risk mitigation strategy at a specific place, for a given detailed development plan, requires measures to be taken outside the physical area covered by the plan. Such measures may mean that the effects of the risk are moved to other locations, for example, the rerouting of traffic. It is very difficult and complicated, both administratively and practically, if the kind of measure required has not been prepared in advance, e.g. in master planning. It may be necessary for different parties at local, regional and national level to reach agreements. In some cases, measures that would provide an optimal solution from the local perspective may be difficult or impossible to realize in a specific project due to regional or national considerations. Clearly there is also the risk of sub-optimisation if different risk management strategies are applied along the same route, without taking the whole picture into consideration.

\subsection{Conflicts of Interest between Transportation Authorities and Developers}

There are basic conflicts of interests between the needs and objectives of infrastructure managers (roads and/or tunnels) and the local authority in charge of development, or another local party such as a developer, wishing to develop the land over or adjacent to the transportation route. The infrastructure manager will want to avoid restrictions on the type and amount of dangerous goods that can be transported, while the land owner will want to avoid development restrictions in order to maximize the development potential. A crucial factor in overbuilding projects is how this conflict of interests is dealt with in the development and application of risk-reducing measures. If no restrictions are placed on the dangerous goods or land use, there is an evident risk that this will result in a lack of risk control. This may give rise to a critical uncertainty as we do not know what kind and frequency of dangerous goods transport will be necessary in the future. Depending on the tolerance deemed necessary, the prerequisites for the design and use of the overbuilding are can be significantly impacted and therefore compromise the project business case. Overestimating the requirements for future transport of dangerous goods will lead to an unnecessarily expensive construction, or underuse of the facilities, for example, if the number of apartments built is restricted. On the other hand, if the future needs for dangerous goods transports are underestimated, then the level of risk will be higher than deemed acceptable by society, or it may be necessary to repurpose the over site development, which can be cumbersome and rarely done. It is possible to predict the needs of dangerous goods transport a few years hence, but this kind of development is expected to have a technical lifetime of 100-150 years.

\subsection{Uncertainties may Lead to Future Surprises}

If decisions are rushed, and inadequate solutions are implemented, or if conditions change in unpredicted 
ways, it can cause problems. The overbuilding is expected to be in use for many years, and there is little scope for changing the nature of the risk or the risk mitigating strategy after the overbuilding and over site development has been completed, and then only by a small number of parties. Unfortunately, the acceptable level of risk may be exceeded without our being aware of it, as there is no regular control or supervision of the amount of dangerous goods transported. This means we will have constructed a potential "black swan", at least for some stakeholder. This phenomenon has been the subject of discussion in risk research in recent years (for further details see Ref. [10]). In the present context, a black swan is a surprising extreme event relative to one's knowledge/beliefs. These can be classified into three different types: (a) unknown unknowns; (b) unknown knowns (we do not have the knowledge but others do); and (c) events that are judged to have a negligible probability of occurrence and are thus assumed not to occur. All three types may arise in the present type of development.

A black swan is the kind of risk that, in most contexts, we want to avoid, or protect ourselves against, but which is perceived as being difficult. As this kind of risk is difficult to imagine before it happens, it is difficult to prepare for it or deal with it. Research in risk in many areas involves developing strategies to identify black swans, and methods of managing this kind of risk. Therefore, when creating overbuildings, we run the risk of creating black swans for future generations, by inadequate risk management. This is controversial and hard to align to sustainable development if not dealt with.

\section{Analysis of the Nature of the Risk}

To provide a more detailed picture of the nature of the risk related to transport of dangerous goods and overbuildings and the requirements for risk evaluation, a number of important factors are analysed, based on an inventory and study of recent research and experience in other areas.

\subsection{Risk Exposure above the Overbuilding}

People in the vicinity, or on top, of the overbuilding covering a transport route for dangerous goods can be exposed to risk from an accident inside or outside the tunnel, mainly at the tunnel portals. People near ventilation openings, including tunnel ventilation shafts, may also be exposed following accidents in the tunnel. Both these types of risk exposure have been relatively well investigated and proven measures are available.

The kind of risk that gives rise to the greatest problems in risk assessment and control is the transport of goods that could result in explosions. One of the complicating factors is that it is practically and economically impossible to design the overbuilding so that it can withstand the maximum permissible load weight of explosives, i.e. 16 tons. As a result, an accident can lead to an unacceptably high risk in an overs site development where the density of people within the area affected is limited due effects of progressive collapse. In previous projects (see e.g. Ref. [11]), it has been deemed to be technically and economically feasible to design a tunnel with over site development, such that damage in the event of an explosion of 1-2 tons in the tunnel is severe, but the impact to the apartment buildings above is limited. If a development is designed for such blast loads, and an explosion equivalent to 16 tons occurs, it is estimated that the damage to the tunnel will be so severe that the buildings on top would collapse. Guidelines have been developed for the construction of air-raid shelters and similar buildings that can withstand explosions of considerably higher force, but the basic principles for this kind of construction are not compatible with a road tunnel with an overbuild. It is thus not possible to construct the tunnel and the development to withstand this kind of accident with only limited damage. Unfortunately, loads of this size are not uncommon when explosives are transported, even though the frequency of transports can be relatively low $[12,13]$.

The kinds of goods that can cause such scenarios are 
explosive materials (ADR Class 1), oxidants and organic peroxides (ADR Class 5). Accidents with combustible gases (ADR Class 2.1) such as LPG and LNG, can lead to gas explosions consisting of a rapid increase in pressure as a result of combustion of the gas/air mixture. Such an explosion usually results in so-called deflagration, i.e. a high-pressure shock front, but lower than that resulting from a detonation. There is a theoretical possibility that a gas explosion in combination with high turbulence could lead to detonation, but this has not yet been observed in a road or rail tunnel. This means that, in practice, it is possible to construct a tunnel able to withstand an explosion involving a Class 2.1 substance.

Although the transport of large amounts of explosive goods can be expected to be limited, an accident in which buildings on top of the overbuilding are affected cannot be ruled out unless transportation is restricted. The probability of such an accident is low, but still possible. The possibility of serious consequences, i.e. resulting in a large number of fatalities, cannot be excluded. The worst case scenario for a single truck involves the explosion of 16 tons of explosives, and it is not practicable to construct the overbuilding to prevent damage to buildings on top of it. The extent of the residual risk is determined by the structural damage, as well as the number of people affected. This will depend on the extent of the overbuilding and the type of over site development. (For further information on accident scenarios the reader is referred to the extensive study presented in the report for the Northern Link project at Hagastaden in Stockholm [11].)

\subsection{Comparison with the Open Road Network}

Extensive damage and injuries can also be expected as the result of an explosion on the open road, but there are a number of important differences between this and an explosion under an overbuilding, which will be discussed below. Firstly, the pressure build-up in a confined space such as a tunnel will be considerably greater than on the open road. The effects on the tunnel and the foundations of the buildings above will thus be much higher. Another important difference is that the probability of an accident resulting in an explosion is higher in a tunnel than on the open road because trucks carrying dangerous goods can affected by a fire in number of another vehicles in the tunnel, which possibly increases the probability of such an accident.

\subsection{Presentation of the Risk}

The effect of an accident in a tunnel on the development above can be simply expressed using F-N curves. This provides information on the spread in probability and the consequences of the various scenarios that together represent the total risk. The method has been thoroughly described in the literature (see e.g. Refs. [14, 15]). A common way of expressing and presenting acceptance criteria in such a graph is to use the ALARP (as low as reasonably practical) region. This is not the only way of presenting risks, but it provides a good way of illustrating the risk contributions from the various scenarios considered. The use of the ALARP region to evaluate the risk has both advantages and disadvantages, but it is commonly used to describe most kinds of major hazards. The ALARP region has been determined to be more suitable for evaluating the risk in this particular case than a pure cost-benefit analysis [14].

Fig. 2 shows an example of the use of the ALARP region for the evaluation of risk in vicinity of dangerous-goods routes (light full lines). The dashed parts of the light lines are extrapolations to the region where risks are not normally acceptable on only a risk-based evaluation, e.g. due to the catastrophe potential. In case of an accident with explosives, it cannot be ruled out that the F-N-curves are close to the grey shaded area due to the magnitude and uncertainty in the probability and the consequences.

\subsection{Potential for Catastrophe}

The potential for accidents in tunnels leading to a catastrophe due to fatalities in the surrounding 


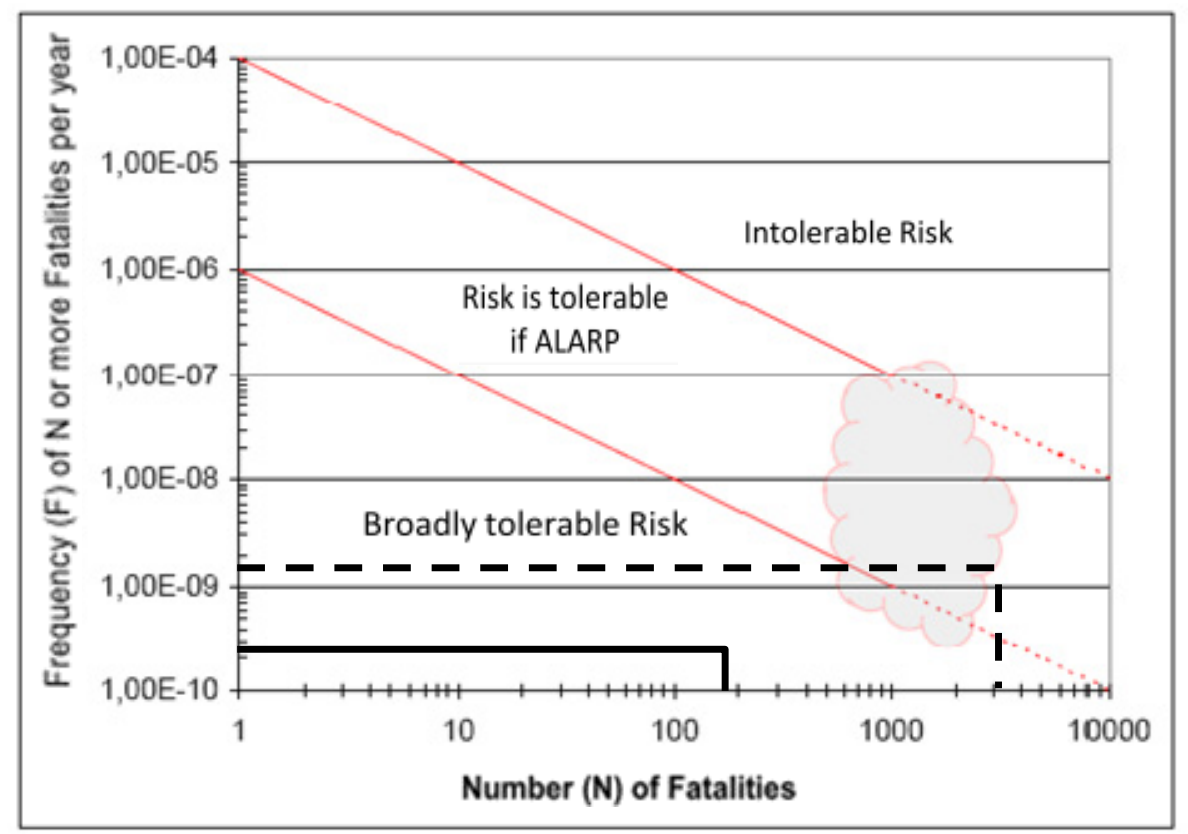

Fig. 2 F-N curves, where the grey shaded area represents a level of risk where the acceptability can be questioned.

buildings may be much higher than on the open road. The extent of material damage and personal injuries may, therefore, be of such a magnitude that society will not have the capacity to deal with them in terms of emergency services and health care. The potential for catastrophe is strongly dependent on the planned land use and development. Risk evaluation is therefore a central aspect in determining the type of land-use, buildings and floor-space index to be permitted on and around the overbuilding.

The potential for catastrophe in a scale where one can question if such risk is reasonable or not, even if the probability is very low, arises if the following three factors coincide: (1) dangerous goods are transported in the tunnel; (2) the construction cannot be designed to prevent structural damage or fatalities in the buildings above the decking for an explosion involving the maximum permissible load of 16 tons; and (3) the degree of development above the deck is high, leading to the risk of a large number of people being affected by an accident.

Such a catastrophic risk is often characterized by high uncertainty, low probability, and very severe consequences. It is far from clear whether the acceptance criteria for these kinds of risk scenarios can be extrapolated according to the light lines/F-N curves in Fig. 2; rather, the opposite. In the criteria developed in several countries, an activity is not permitted if the risk exposure exceeds a certain size, e.g. 1,000 fatalities, based solely on quantitative criteria $[16,17]$. It is stressed that the decision making must be risk-informed rather than risk-based. In the criteria proposed for the chemical industry in Sweden, which are used in many applications, it is clearly stated that: "No upper limit for the consequences has been proposed as part of the criterion for societal risk". This kind of issue should be addressed qualitatively [15]. The question remains as to how this should be done. In practice, very little attention is paid to this at the present time.

One of the main threats to overbuilding projects described at the beginning of this paper is illustrated by the bold dashed line in Fig. 2, i.e., a risk scenario with very low probability but very severe consequences. Another situation that can cause a similar threat is if the risk analysis results in a risk profile represented by the bold line in Fig. 2, but uncertainties are present that can shift the risk profile towards the bold dashed line. The 
representation of the risk profile by the lines is highly dependent on the assumptions and limitations applied in the underlying risk analysis. Examples of these are whether explosive materials are transported along a certain route, or if only limited quantities are transported, for which the tunnel and over site development is designed. The development above the decking can also be restricted so that the number of people that can be affected at the same time is limited. In such cases, there is a good possibility that the residual risk will not exceed what is considered to be acceptable. The dilemma arises when the size of the design blast load is derived from the size of the cargo load and number of transports based on current conditions, and there are no restrictions on future loads. If the maximum permitted load and/or frequency should increase in the future, then the risk would extend into the region indicated by the grey cloud.

\subsection{The Extent of the Risk}

One of the questions that arise is whether the level of the acceptable risk is correct. Shouldn't the benefit to society allow a higher level of risk than that applied in other kinds of development projects? A brief assessment of the risk profiles arising from substances that can cause an explosion (mainly ADR Class 1 and Class 5) shows that the average risk in terms of expected fatalities per year is very low, far below the average risk from other hazards not exceeding the acceptance criterion. This means that the problem does not lie in the fact that the proposed development would lead to a higher number of expected fatalities per year than in other kinds of developments. The individual risk does not pose any problem either, as the probability of this kind of accident is generally very low. Based on experience from a number of projects, it is deemed very probable that for most of the buildings above the decking, the individual risk without any protective measures will be lower than that accepted in other contexts, e.g. development with buildings within $100 \mathrm{~m}$ of a railway or housing estates near airports. The problem, as mentioned above, is the severity of the consequences associated with a very serious accident.

This means that the problems associated with decision making and risk management would not be resolved by increasing the acceptable risk level. This would not lead to the acceptance of a facility with a risk profile that borders on the grey shaded area in Fig. 2. To achieve such an alleviation it would instead be necessary to increase the acceptance of catastrophes - not unlike the kind caused by the tsunami in Fukushima in Japan in 2011. This would mean the deliberate construction of a facility with the potential for a catastrophe of such dimensions, for future generations. The effects of the tsunami in Fukushima have been discussed in the scientific literature from many perspectives. It has been questioned why the protective walls were not designed to offer protection against the highest previous tsunamis in the area, rather than being satisfied that the height chosen was adequate according to design praxis [18]. The same applies to overbuildings and how much foresight we need to have is unclear.

\subsection{Large Uncertainties}

For obvious reasons, few empirical data are available on the effects of explosions in tunnels in the urban environment. There are considerable uncertainties in the estimation of the probability and the extent of the consequences. However, the physical effects, in terms of pressure, temperature and vibrations, are relatively well known, and both simple and more complex models are available to study the phenomena. Despite the fact that these models are based on simplifications and assumptions, they provide useful information for the assessment of the extent and severity of such accidents. It could be argued that the tools are still too crude, but in general, they are quite well developed, and this particular kind of accident is not exceptionally problematic compared to other decision-making situations associated with risk management. The uncertainties involved are 
considerably lower, for example, than in the assessment of the effects of solar storms on IT infrastructure, or the spread of pandemics in society. However, it is important to understand there are uncertainties, and that attention is drawn to them so that decision makers are made aware of them. A number of sources of uncertainty have already been mentioned above, but a summary is given below of the parameters associated with uncertainty that will affect the probability of an accident of the kind considered here:

- Transportation and future traffic developments;

- Transportation patterns (which are affected by developments of the road network and possible future restrictions);

- Regulations governing transportation, market conditions and authorization for the production and use of hazardous materials that can cause explosion;

- Political decisions the affect the proportion of traffic on roads and on railways;

- Cargo capacity, i.e. increased allowances in cargo capacity;

- Economic conditions that affect distribution patterns including transportation costs;

- The location of production plants and distribution centres for explosive goods;

- The number of purchasers/users of explosive goods;

- The state of the economy (the transport of goods is strongly correlated to the GNP);

- Security risks and antagonistic threats;

- The enforcement and compliance of restrictions.

Even if we have some idea of how goods are transported today, conditions will change with time in a way that we cannot predict or control at present. This means that the uncertainty associated with future transportation patterns and size and frequency is high. This constitutes an intrinsic uncertainty that affects not only the nature of the risk, but also our ability to evaluate it. The only way to reduce this uncertainty is to ensure that the residual risk is not exceeded, even when the maximum amount of explosives is transported. This amount of goods can be reduced by tunnel restrictions so that it is possible to reduce the risk by designing the tunnel construction to withstand an explosion.

Uncertainties, and the way in which they have been considered, as well as the consequences of not taking them into account, thus constitute an important component in the information on which decisions regarding risk are based.

\subsection{Low Probabilities}

It is sometimes argued that when the probability of a certain kind of accident is extremely low, it can be disregarded. But on what basis can we define a negligible probability or risk in relation to a particular scenario? We need to be careful about ignoring scenarios on the basis of isolated risk and probability assessments, as even very unlikely events may occur [19].

Explosions are rare in the transport sector, but there are examples of serious accidents (see, e.g. Ref. [20]). One such accident occurred at an open-cast mine in Norway in 2013, when a truck carrying 15 tons of explosives exploded on the surface road. The driver was miraculously uninjured. Other examples can be found in Refs. [21, 22]. If an increasing number of urban tunnels are constructed, each with a negligibly low probability of an accident, the overall probability of an explosion in such a tunnel will steadily increase. Serious industrial accidents are characterized by a series of unfortunate circumstances, where the probability of a single event may be very low. According to Perrow (and Aristotle) [23], "It is probable that the improbable will happen". One can, therefore, not simply ignore the potential for such accidents merely because their probability is low. From the point of view of decision-making theory, the weight that decision makers place on fear and uncertainty, and the weight afforded serious consequences in relation to minor consequences, will be decisive in risk evaluation. The monetary value placed on human life will 
ultimately determine which measures are deemed necessary to reduce the risk to an acceptable level.

\section{Dealing with the Problem of Risk Control}

Maybe a little bit contradictory, decking and overbuilding is generally an effective risk-reducing barrier, e.g. for existing buildings near the tunnel. However, problems arise when dangerous goods are transported through the tunnel, combined with a high density of people in the direct vicinity. Analysis of the nature of the risk also shows that in such situations: risks that are not dealt with can lead to a catastrophe, albeit with a low probability; that the uncertainties associated with the risk are high; and that implementing risk-reducing measures that allow risk control is complicated. Recommendations can be developed by comparison with other so-called major hazard industries (e.g. nuclear power, chemical, off-shore, dams in power industry, etc.) and a survey of recent research into risk analysis in this area.

\subsection{Strategies for Risk Management}

The problems and frustration experienced in real projects seem to be due to the belief that it should be possible to solve problems by so-called risk-based decision making. A quantitative risk assessment produces values expressed as probabilities and expected values that are compared to acceptance criteria. Such a risk management strategy is suitable in cases where there is sound knowledge and the uncertainties are small, which is not the case with overbuildings, mainly regarding future transports and severe damage. This appears to be a fundamental reason why no progress is being made in practical projects in this area. A growing number of researchers and analysts have found probability-based approaches to understanding risk to be too narrow [24]. The main reasons are:

- Assumptions can conceal important aspects of risk and uncertainties;

- The probabilities can be the same, but the knowledge on which they are based may vary;

- The approaches are often based on historical data;

- Surprises occur (black swans);

- There is too much reliance on probability models and frequentist probabilities; and

- Probability is just one of many tools that can be used to describe uncertainties.

In other words, uncertainties are not properly reflected when using this risk assessment procedure. Uncertainty assessments extending beyond probabilities and expected values are necessary in making informed decisions on the acceptability or tolerability of risks [25]. Since technical risk analyses represent a narrow framework, they should not be the sole criterion used for decision making regarding risk [26]. Thus, different foundations are required in decision making for the management of catastrophic risks that complement traditional decision analysis and expected utility theory [27].

The strength of the knowledge on which the probability assessments are based is not reflected in the probabilities used in comparisons with acceptable levels [28]. The crucial question here is what degree of uncertainty and ignorance the main stakeholders and public interest groups are willing to accept or tolerate in exchange for a potential benefit. The use of such criteria can easily lead to the wrong focus, namely meeting the criteria, rather than finding the best possible solutions and measures, taking into account the limitations of the analysis, uncertainties not being reflected by the analysis, and other concerns important in the decision-making process.

As a reaction to risk-based decision making, various risk management strategies or approaches have been developed with emphasis on different complimentary aspects. Research initiatives highlight three approaches that are commonly used to manage risk: risk-informed, cautionary/precautionary, and discursive strategies [26, 29, 30]. Emphasis is put on arriving at a suitable risk management strategy by combining three pillars according to the nature of the risk: 
- The use of risk assessment;

- Robustness, resilience and cautionary principles;

- Building trust and participation in the decision-making process.

In order to achieve this, it is necessary to balance the risk-informed, cautionary/precautionary (robustness, resilience, adaptive) and discourse-based approaches. This is the general approach and is fundamental for risk management. The results of quantitative risk analyses must be considered in relation to the assumptions made and the knowledge on which the analyses are based [10]. The dimension uncertainty, knowledge and surprise in risk analysis must be adequately reflected, but this has not been addressed until recently.

A risk assessment could provide useful decision-making support, although emphasis must also be placed on the uncertainties. According to the literature (e.g. Refs. [31, 32]), the precautionary principle reflects the idea that if the consequences of an activity could be serious and are subject to scientific uncertainties, then precautionary measures should be taken. In extreme cases, the whole activity should be forbidden or terminated. So the critical criteria used to establish whether the precautionary principle should be applicable in the present case is both the possibility of catastrophic outcomes and the existence of scientific uncertainties. It is stated in the literature that the ALARP region is in line with this concept, i.e. cautionary approach, but that traditional CBA (cost-benefit analysis) and the expected value are inadequate [28].

The so-called precautionary-based strategy is suitable, when scientific uncertainty is so significant that precautionary or adaptive measures are required. In other cases additional risk reduction measures are sufficient. A detailed risk assessment providing predictions and characterization of the uncertainties can provide input for such a judgement of suitable strategy. The more uncertain the consequences are, the more decision makers are driven to apply the precautionary principle even if the expected value for each risk dimension is rather low [25].The above reasoning can be exemplified by the decision of the County Administrative Board of Stockholm to apply restrictions on the transport of dangerous goods that could lead to serious explosions along parts of the Northern Link tunnel in Stockholm [33].

\subsection{The Management of Uncertainties}

Two important conclusions can be drawn from the survey of recent researches that differentiate the suggested approach from the traditional risk-based approach. The first is that the uncertainties themselves are an important part of the information on which decisions are based, and affect the decisions made. The other is that part of the risk management strategy involves taking measures to reduce the uncertainties. It is acknowledged that risk analysis as a tool has limitations and robust approaches are required to deal adequately with uncertainties. The PSA-N nuclear regulations state, for example: If there is insufficient knowledge concerning the effects that the use of technical, operational or organizational solutions can have on health, safety or the environment, solutions that will reduce this uncertainty shall be chosen [28]. This strongly indicates that efforts must be made to reduce uncertainty, and not only the risk. In the same manner, measures must be taken to increase knowledge, without necessarily reducing the risk.

\subsection{Assumptions}

Several risk analysis researchers have highlighted the need to consider the results of risk assessments in the light of the assumptions made [34, 35]. Another important aspect closely linked to the strength of the knowledge available is the underlying assumption applied in the analysis. Aven [36] proposes the following framework. First, the assumptions must be identified and listed. This task in itself is challenging. The next task is to perform a qualitative risk assessment of these assumptions, highlighting and presenting deviations from these statements 
(assumptions), the implications of such deviations, judgements of the probabilities and the strength of the related knowledge. Attention should also be paid to questions such as: "Do we understand the phenomena involved?", "Do we have evidence supporting our judgements?" and "Have our beliefs been checked by others?".

The first stage of the analysis should include the following elements in particular: (a) a qualitative assessment of the information value of the metrics in relation to informing the decision maker about risk; (b) a qualitative assessment of the risk related to deviations from the assumptions made (this assessment covers deviations and the effects of these deviations, probability judgments, and strength of knowledge judgements (see c); and (c) a qualitative assessment of the strength of the knowledge.

\subsection{The Strength of the Knowledge}

The need to link risk with knowledge is also highlighted. This is a relatively new concept, but has nonetheless been investigated by, for example, Aven and Zio [24]. Risk descriptions are not objective, but are developed based on data, information and justified beliefs. This basis is analyst dependent, so even if the information on which it is based is the same, this will not necessarily lead to the same risk descriptions by two different analysts. There is no way of ensuring that two assessors having the same background knowledge would assign the same probabilities [15]. Hence there is no alternative that allows for some type of process that can see beyond the quantitative results of a risk analysis. The values obtained provide information for the decision maker, but do not prescribe what to do.

One way of making use of knowledge is to divide it roughly into strong and weak knowledge, and report these separately so that decision makers can take this into account. The dimension of knowledge, at least, should be taken into account in the choice of measures. For example, if knowledge is limited, the decision could be made to acquire better knowledge before any decision is made. Various ways of doing this have been reported., e.g. by Aven [37], who presents a crude method of incorporating the strength of knowledge in decision making using the ALARP principle, which can be regarded as a relatively straightforward tool that can be applied in practice.

\subsection{Surprises}

Another concern is the common use of probabilities. Although the aim of a probability is to express the uncertainty or variation in relation to future situations, it is often used simply as a function of historical empirical data. Thus, important aspects of change and potential surprise are not taken into account. Furthermore, if the probability of a certain event is calculated from a finite amount of existing data, this may have little value in predicting the probability of a future event. A checklist is provided by Bjerga and Aven [13], highlighting potential surprises relative to the analysts' knowledge to provide a good starting point for the decision maker to analyse the situation in question.

(1) The possibility of unknown knowns. Have special measures been implemented to check for this type of event (for example, an independent review of the analysis)?

(2) The possibility of events being disregarded due to their very low probabilities, although these probabilities are based on critical assumptions. Have special measures been implemented to check for this type of event (for example, signals and warnings influencing the existing knowledge basis)?

(3) Risks related to deviations from the assumptions made.

(4) Changes in knowledge over time.

The reason for addressing surprises is to obtain a stronger focus on issues not covered by the traditional risk perspectives, which are based on historical data, probabilities and expected values. At the same time, it is crucial to address known events in the risk analysis whose probability of occurrence is judged negligible, 
and are thus not believed to occur.

\section{Nuancing of Risk Control}

The conditions affecting risks can vary considerably, e.g., between high-density through traffic on an urban motorway and local traffic on smaller roads. Knowledge of size and frequency of expected future transport may vary for different kinds of roads at different locations. Examples of this are a secondary road for transport of dangerous goods where a consumer in terms of a local industry only has a permit for the handling of certain dangerous goods types compared to a dual carriageway used as thoroughfare. Another example is if a primary route intended for the transport of dangerous goods, although it is mainly used by local traffic, and no dangerous goods, or at least explosive goods, are transported and there are no producers or consumers of dangerous goods. It is therefore important to address the possibility of the adaptation of protection by using risk analysis approaches specially adapted to the specific situation. Bearing in mind the construction in question, an attempt is made below to nuance risk control in three cases, based on the analysis of the nature of the risk, and the tools available for risk control presented in the previous section. These cases have not been studied in detail, but are intended to illustrate the potential of this approach. The conditions for risk evaluation and the risk management strategy, and thus for risk control, differ in these cases, which are denoted: "off the scale", "potential black swan" and "low-risk".

\section{Case 1: The off the scale case}

This case exemplifies high-density traffic on an urban motorway, classed as a primary route for the transportation of dangerous goods. The stretch of road in question is used by both through traffic and local traffic. The nature of the risk can be influenced by applying risk-reducing factors, as shown by the bold dashed line in Fig. 2. Risks can, in general, be reduced by measures that reduce the probability or the consequences of an accident, or a combination of both.
Two possible strategies can be identified to evaluate the risk described above: by reducing the consequences to the extent that the level of risk is inside the boundaries for established risk criteria, or by accepting a low probability of a catastrophe.

\section{Consequence-reducing measures}

From the perspective of traffic safety in tunnels, probability-reducing measures are usually given high priority. It has been made clear in the European regulations for railway tunnels (TSI, [38]) that this is a basic component in the development of safety concepts in tunnels. Examples of measures taken in road tunnels are reducing speed limits, prohibiting overtaking, and installing safety systems such as sprinklers in combination with a drainage system. Sprinklers significantly reduce the probability that a fire in a tunnel, for example, in a truck, will spread and involve other vehicles transporting explosives. Despite the positive effects of sprinklers, they will not solve the problem of risk evaluation in the present case, as the risk of catastrophe would remain, even if the level of risk were reduced. Consequence-reducing measures should therefore be considered in addition. The consequences can be reduced by designing and adapting the tunnel (overbuilding) and the development on top of the tunnel, and by restricting the kind of traffic allowed in the tunnel. The choice of measures is strongly related to the way in which conflicts of interest between the infrastructure manager and developers are dealt with.

The design of the tunnel

Risk reduction can be achieved through a combination of measures to increase the resistance of the structure to the effects of an explosion, e.g. by limiting the effects of a blast through the decking, or reducing the risk of progressive collapse. This may call for measures to be taken in the construction of the overbuilding, its foundations, and the overlying buildings.

\section{Traffic}

The type of goods transported in the tunnel can be 
restricted, but his means that it must take other routes. Restrictions can be designed so that they apply only at certain times of day. For example, only allowing the transport of certain types of dangerous goods during the night-time, when shops or offices built on the decking are empty. Most dangerous goods can be transported through the tunnel at any time, even during the day, provided the restrictions are adapted to the design load of the construction works or vice versa. It may then be necessary to provide a secure base for trucks carrying dangerous goods in the vicinity of the tunnel.

The use of tunnel restrictions places high demands on a holistic approach, as these measures may have effects outside the geographical boundaries of the detailed development plan. Risk exposure will be moved from one location to another. Also, the transportation needs of the producer and consumer must be met, which may necessitate measures elsewhere. Other ways of influencing traffic and/or the implementation of restrictions include the provision of new routes, the supply of goods from another location, or the relocation of businesses or industries. Another important aspect is the enforcement to take place.

During the operation of a tunnel, it is generally necessary to temporarily divert traffic along a route used for rerouting during renovation etc. This route can sometimes also be considered as an alternative route if restrictions are applied. In any case, it will be necessary to consider the risk exposure along the new route, which requires an overall assessment to determine whether it is suitable or not. Examples of frameworks for such assessments have been presented previously [39]. As the exposure to risk above the tunnel will be mainly affected by explosions, it is possible to adapt the restrictions so that they only cover classes of dangerous goods that can result in powerful explosions. It is also worth pointing out that those restrictions generally only apply to a small proportion of the transports. Such restrictions on the transport of goods that can cause serious explosions are available according to the ADR classifications, i.e. if a tunnel categorized as a B-tunnel. From a societal viewpoint the impact on transport work should have little or no effect, but should be considered in each individual case.

\section{Buildings}

The kind of buildings constructed on top of the decking should be determined by the nature of the risk. Risk reduction measures will affect the type of buildings as limitations will be placed on their design, type and volume. This may make the building of car parks or shops more suitable than apartments, as the former are generally only occupied during the daytime. The desired effect of measures is to limit the number of people simultaneously exposed to a risk. If a multi-story car park is required in the area, it could be built on top of the decking, as could a recreational area or a local road.

An extreme example of a measure is not to allow any kind of buildings on the decking. Such a measure would naturally affect the potential for development, but land would still be made available close to the tunnel. It should be noted that considerable demands may still be made on the construction to ensure that it can withstand an explosion of a certain force.

\section{Accepting the potential for catastrophe}

Another way of dealing with the "off the scale" situation is to consider reducing the probability of an accident as far as is reasonably practicable, and then accepting that there will be a low probability of an extreme event occurring. The knowledge base is often weak, and the probabilities are subjective (judgmental, knowledge-based), and more or less strongly founded. There is no clear correspondence between the probability assignments and the actual occurrence of the events, and it is therefore necessary to scrutinise the judgements concerning acceptable risk and negligible probability, and the background knowledge on which these assessments were based. For this alternative to be possible, important assumptions and uncertainties must be clearly presented in the original analysis. 
There are examples of areas where this kind of approach has been applied, mainly in cases of bursting dams and landslides, where the approach has been formalized [16, 40]. For example, the tolerability limit for the maximum number of fatalities (arising from a single event) should be increased from 1,000 fatalities to 5,000 fatalities (see Fig. 3).

Vertical lines are drawn in the F-N graph in Fig. 3 at 1,000 fatalities and 5,000, up to the line, above which the number of fatalities is unacceptable. The region between 1,000 and 5,000 fatalities is regarded as an "intense scrutiny" region. According to Aven [10], there is no clear correspondence between the probability assignments and the actual occurrence of the events. Hence, it is appropriate to scrutinise the judgements concerning both the acceptable risk and negligible probability, and the background knowledge supporting these judgements. Therefore, such a scrutiny must be based on the following acknowledgements: that the acceptable risk should not be determined by a judgement of probability alone; that events may occur, even if the probabilities is very low; and that cautionary and precautionary principles constitute essential pillars of the risk management associated with such events. The reason for including an "intense scrutiny" region in the risk guidelines is to provide regulators with the option to permit certain types of developments. Such developments may not necessarily be associated with an unacceptable level of risk, but could be examined with special scrutiny bearing in mind societal needs and additional requirements that reduce the probability further.

As it has been possible to formulate this kind of acceptance criterion in other areas, it is not impossible that this may be applicable to the case of overbuilding, bearing in mind that the benefit of the development to society is very high. However, this would be controversial in Sweden, as we have no general tradition of this type of application of risk evaluation and no application has been found for overbuildings in

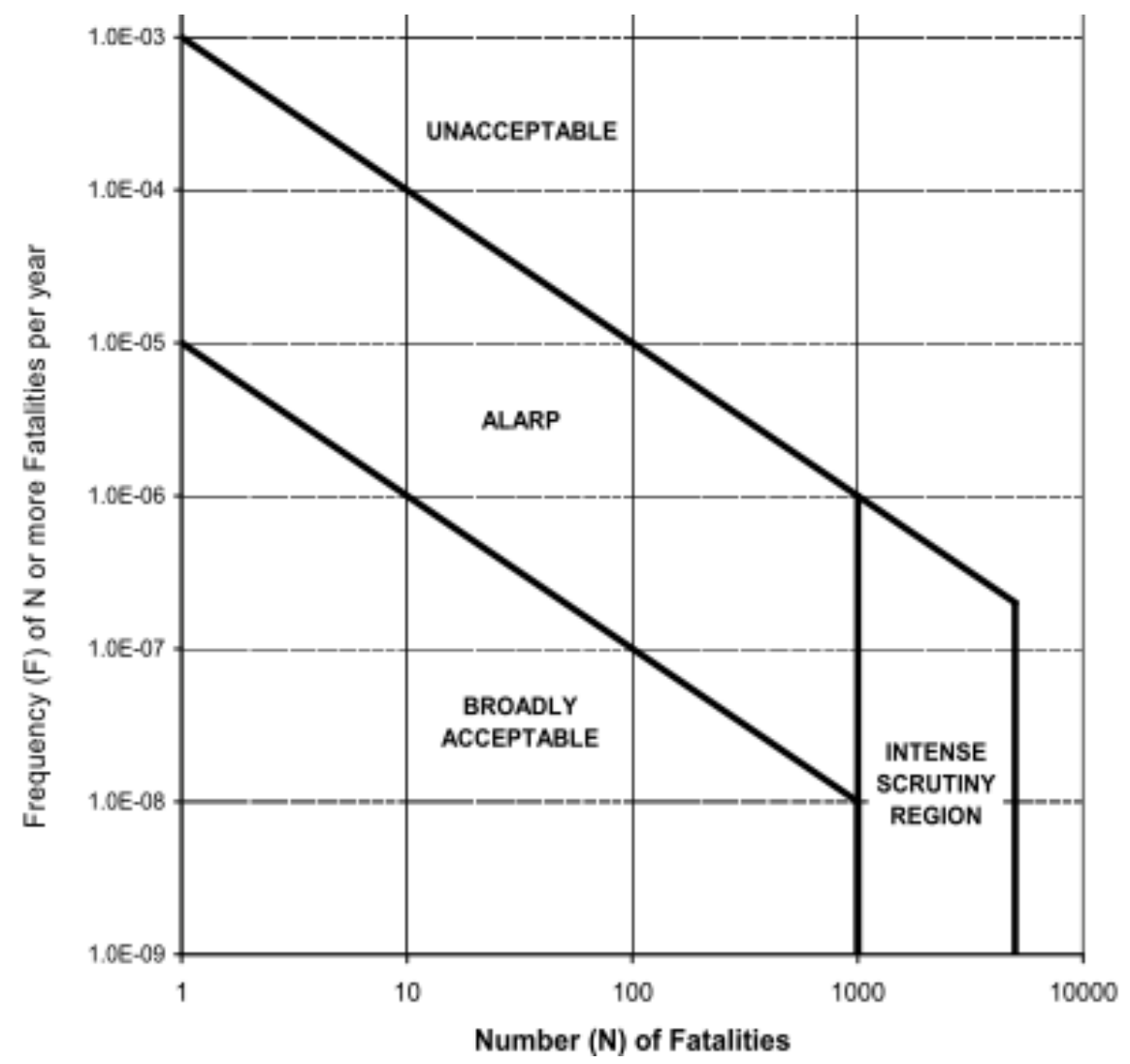

Fig. 3 Example of an F-N curve for evaluating the societal risk for in the case of landslides. 
any other country. Such an agreement should therefore be carried out using a discourse-based risk management strategy among relevant stakeholders and authorities.

\section{Case 2: A potential black swan}

This case is exemplified by overbuilding of a stretch of secondary road or a primary route without through traffic or local producers or consumers of explosives. Under such conditions, the uncertainties will be smaller, and knowledge concerning the goods being transported is better, as it will be based on the needs of local producers and consumers. The challenge lies in the uncertainty in how explosive goods will be transported in the future. The nature of the risk and its challenges is characterized in Fig. 2. This should be suitable for situations where the risk-based on the planned construction and the predicted transport of dangerous goods does not exceed the acceptance criteria, but where unexpected developments in transportation lead to uncertainties in the judgements, resulting in it being impossible to exclude the potential for catastrophe.

\section{A robust solution}

One way of achieving risk control is to take the corresponding measures from the "off the scale" case, but this may involve unnecessary limitations and restrictions, or less than optimal exploitation of the opportunities offered by overbuilding, i.e., a waste of public finances. However, this provides a margin for future changes and a robust solution, and is not depending on monitoring or control.

\section{An adaptive solution}

An adaptive solution should also be possible for the construction in question. In this case, risk management should be directed towards having control over the risk in order to be able to take measures, e.g. restrictions, only when the amount of dangerous goods being transported approaches the limit for an acceptable level of risk. This kind of risk management places demands on dialogue and cooperation, and requires that good knowledge be maintained, for example, by monitoring the amount and type of goods transported and/or taking an active part in the planning and processing of applications that affect the kind of goods transport in question. This means that future planning and processing of applications should be connected to this strategy. Future handling and transport of dangerous goods can be supervised and controlled in connection with the issuing of environmental permits and authorization for the handling of combustible and explosive goods. Regulations and mechanisms for controlling such goods are available, but are not yet used for this purpose in local planning and authorisation. Methods must be developed to use this knowledge and risk control instrument correctly in the management of the kind of risks discussed here. For example, environmental permit to operate should not be granted for a business if the transport of explosive goods cause negative effects or another location of the business might be necessary to consider in city planning. Some initial thoughts on this are presented in the final report on the project Urbana Stråk in Stockholm [12]. Demands are also placed on techniques for the monitoring of the transport of dangerous goods. Techniques have been developed, but are not in regular use today. It is also necessary to have a "Plan B", in case future developments are not as expected (i.e. a surprise). One way of ensuring this could be to make provision for a future re-categorisation of the tunnel, if and when necessary, by planning an alternative route and ensuring that the risk exposure along this route could be managed before the route is taken into operation.

\section{Case 3: The low-risk case}

The third alternative is characterized by stretches of road in urban environments on which no dangerous goods, or at least explosive goods, are transported and will never be. This should be the most common situation in large cities. In such cases, overbuilding will not be a problem, and can be regarded as a risk-reducing measure. This is a completely different case to that described in this paper. Overbuilding of such routes should be considered, in the first place, 
when the aim is cost-effective solutions with low project risk.

\section{Conclusions}

Overbuilding in urban environments should, in general, not pose problems, notwithstanding the risks for tunnel users. An exception is in rare cases when applied to routes used for the transport of dangerous goods, together with overlying developments in which large numbers of people are simultaneously exposed to risk. In such cases, challenges are unavoidable. Knowledge and the results of research on the management of major hazards and other risks that have been obtained in recent years are not fully exploited when considering overbuilding of dangerous-goods routes. There is thus a considerable potential to make better decisions and reduce the risks associated with future overbuilding projects. This knowledge can also serve as the basis for the development of practical tools.

Knowledge concerning conducting risk analysis and a lack of data appears to not be a serious problem. Neither is it necessary to perform more extensive risk analyses in order to evaluate the risk. If anything, a better, more adapted risk management strategy should be developed for the matter in question. Information on which decisions are based should be improved, for example, by shedding light on the strength of knowledge, uncertainties, surprises and assumptions used in the analysis, to give decision makers a better understanding of the nature of the risk. Furthermore, it was found that concrete measures, other than generic risk-reducing measures, may be necessary to deal with these dimensions, e.g. to increase knowledge and/or reduce uncertainty.

Two critical issues were identified regarding risk evaluation and risk control, which concern two fundamentally different situations. The first issue concerns whether it is justified to accept the risk of an accident with a very low, but finite, probability that may result in a very high number of fatalities (e.g. $>$
1,000). The effects of an accident involving the maximum amount of explosive goods that can be transported cannot be adequately reduced by building an explosion-proof construction. It is possible to deal with a relatively high blast load ( $\sim 2$ tons), but not the maximum (16 tons). In order for the risk of a catastrophe to be acceptable, agreement must be reached on suitable criteria for determination of impact significance, i.e. acceptable risk, with the County Administrative Board on the implementation of measures to reduce the probability of such a catastrophe as much as possible. Also, application of protective barriers is necessary to reduce the damage and injuries that could arise from accidents involving most of the transportation of explosive goods. It will still be difficult to determine which, and how many, measures and protective barriers are reasonable. To the best of the author's knowledge, no such risk has yet been accepted in practice.

If the risk of a catastrophe is not accepted, then the main risk management strategy should be the reduction of the consequences. Examples of this include restrictions on transportation, possibly combined with protective barriers, or that the development on the overbuilding and the area surrounding it is designed such that the risk of serious consequences is acceptable (for example $<1,000$ fatalities). Other technical means of reducing the consequences are possible, but will lead to limitations on the development or the transport of goods, which may be costly and difficult to implement.

The other critical issue is how risk control can be ensured when the transport of explosive goods is not currently envisaged, but cannot be excluded in the future. If the risk evaluation does not take into account possible future transport of such goods, a so-called precautionary approach will be necessary. This requires measures such as measuring and supervision of dangerous-goods transport in order to determine the margins, and whether further measures are necessary. Techniques and means of doing this are available. Attempts have been made to do this in pilot projects, 
but there are currently no demands for this kind of survey. It may also be necessary to monitor and assess the way in which future development plans and environmental permits affect the need for goods transport. Continued development and testing in this area can help realize developments with cost-effective safety concepts.

The management of both these critical issues will require the cooperation of a number of parties in a way that is not common today. In practice, this means that many challenges will be encountered in the planning and execution of such a development. However, there is no need for further scientific efforts to make this possible, rather deeper cooperation between the public and private sectors, and the expedient application of the knowledge and research results currently available. Sustainable urban development should be based on the realization of developments for which the level of risk can be controlled, despite future changes in use. Such guidance and control is essential.

\section{References}

[1] Swedish Act on Transportation of Dangerous Goods-ADR (SFS 2006:263). Swedish Civil Contingencies Agency.

[2] Swedish Planning and Building Act (SFS 2010:900).

[3] Swedish Environmental Act (SFS 1998:808).

[4] Swedish Road Act (SFS 1971:948).

[5] County Administrative Board of Stockholm. 2012. Overbuildings-A Knowledge Overview. Report 2012:22.

[6] Tyréns. 2012. A Study of Managment of Haz Mat Transports When Overbuilding Roads.

[7] Swedish Road Transport Agency. 2017. The Transport System in Urban Planning. Report 2016:148.

[8] City of Stockholm. 2016. Budget 2017.

[9] ÅF. 2013. Overbuildings-An External Analysis.

[10] Aven, T. 2015. "Implications of Black Swans to the Foundations and Practice of Risk Assess and Mgmnt." Reliab Eng Sys Safe 134: 83-91.

[11] The Swedish Road Transport Agency. 2009. Risk Assessment: Public Consultation for the Project E 4/E 20 Tomteboda-Haga Södra, 0S141207.

[12] City of Stockholm. 2017. Urban Streets-Project report.

[13] The Swedish Road Transport Agency. 2017. PM Risk Road Planfor E10 at Kiruna Center, Kiruna, County of Norrbotten.

[14] Ale, B., Hartford, D., and Slater, D. 2015. "ALARP and
CBA All in the Same Game." Safety Science 76: 90-100.

[15] The Swedish Rescue Services Board. 1997. Evaluation of Risk. R\&D-report.

[16] Geotechnical Engineering Office. 1998. Interim Risk Guideline. Civil Engineering Dept., Geotechnical Engineering Office, Hong Kong.

[17] Porter, M., and Morgenstern, N. 2013. "Landslide Risk Evaluation." Open File of Geological Survey of Canada.

[18] Paté-Cornell, E., and Cox, A. 2014. "Improving Risk Management: From Lame Excuses to Principled Practice." Risk Anal 34 (7): 1228-39.

[19] Aven, T. 2016. "Ignoring Scenarios in Risk Assessments." Relib Eng and System Safe 145: 215-20.

[20] Mainiero, R., and Rowland, J. 2009. "A Review of Recent Accidents Involving Expl. Transport.” J Explos Eng 26: 6-12.

[21] Wikipedia. 2017. List of accidents and incidents involving transport or storage of ammunition. Accessed August 1, 2018. https://en.wikipedia.org/wiki/List_of_accidents and_incidents_involving_transport_or_storage_of_ammu nition.

[22] Swedish Public Service Television (SVT). 2013. "Truck with 15 tons of Explosives Exploded in Norway."

[23] Perrow, C. 2011. Normal Accidents: Living with High Risk Technologies. New Jersey, USA: Princeton University Press.

[24] Aven, T., and Zio, E 2014. "Foundational Issues in Risk Assessm and Risk Mngmt.” Risk Anal 34 (7): 1164-72.

[25] Aven, T., and Renn, O. 2012. "On the Risk Mgmnt and Risk Governance of Petroleum Operations in the Barents Sea Area." Area Risk Anal 32 (9): 1561-75.

[26] Renn, O. 2008. Risk Governance-Coping with Uncertainty in a Complex World. London, Sterling, VA: Earthscan.

[27] Cox., T. 2012. "Community Resilience and Decision Theory Challenges.” Risk Analysis 32 (11): 1919-34.

[28] Aven, T. 2016. "Risk Assessment and Risk Management." European J of Operational Research 253 (1): 1-13.

[29] SRA. 2015. "Foundations of Risk Analysis, Discussion Document." http://www.sra.org/resources.

[30] Klinke, A., and Renn, O. 2002. "A New Approach to Risk Evaluation and Mangnt." Risk Anal 22 (6): 1071-94.

[31] IRGC. 2015. Guidance for Emerging Risk Governance. IRGC report.

[32] European Commission. 2000. Communic from the Comm on the Prec Princip.

[33] County Administrative Board of Stockholm. 2016. Local Road Traffic Regulations for Transportation of Dangerous Goods 01TFS 2016:28.

[34] Beard, A. 2004. "Risk Assessment Assumptions." Civil Eng Environ Syst 21 (1): 19-31.

[35] Paté-Cornell, E. 1999. "Conditional Uncertainty Analysis and Implications for Decision Making." Risk Anal 19 (5): 
995-1002.

[36] Aven, T. 2017. "Improving Risk Characterisations in Practical Situations.” Reliab Eng Syst Safe 167: 42-8.

[37] Aven, T. 2013. "Practical Implications of the New Risk Perspectives.” Reliab Eng Sys Safety 115: 136-45.

[38] Commission Regulation EU 1303/2014. "Safety in
Railway Tunnels." European Commission.

[39] Lundin, J., and Antonsson, L. 2018. "Guidance and Methods for Categorizing Road Tunnels." In Proceedings of ISTSS, 471-87.

[40] Geological Survey of Canada. 2013. "Landslide Risk Evaluation.” Open File 7312. 